\title{
Representación espacial en invidentes estimulados de manera háptica con un dispositivo mecatrónico, dmrei, y con el bastón clásico
}

\author{
Luis B. Sanabria R. ${ }^{1}$ \\ Artículo presentado el 17 de octubre de 2006 y aprobado el 27 de noviembre de 2008. \\ Spacial representation in blind people hapticly stimulated by a \\ mechatronic device and the classic cane
}

\begin{abstract}
Resumen: La manera en que los invidentes se representan el espacio crea la necesidad de desarrollar ciertas habilidades para obtener información del entorno en estas personas. Una forma de obtener esta experiencia es por medio del aprendizaje espacial, el cual se caracteriza por la capacidad para aprender, representar y actualizar lugares y objetivos (Balakrishnan, Bousquet y Honavar, 1998). Con el objeto de analizar estos procesos en los invidentes, se desarroIla la tesis doctoral "Representación espacial del entorno en invidentes estimulados de manera háptica con un dispositivo mecatrónico, DMREl, y con el bastón clásico", resumida en este artículo. El interés del trabajo doctoral se centra en el estudio de la representación espacial del entorno, producida por el efecto de dispositivos tecnológicos que se adaptan al ser humano mediante sus mecanismos sensoriales. Específicamente, hace referencia a la identificación de las diferencias en la representación espacial, en términos de estrategias con el uso de un sistema mecatrónico comparado con el uso del bastón clásico. Los resultados obtenidos muestran diferencias con el uso del dispositivo, por cuanto este elemento consolida una mejor representación del espacio, lo cual se pudo demostrar en el manejo de una variedad de estrategias, en la anticipación perceptiva y en la actualización del espacio, donde los invidentes lograron mejores realizaciones.
\end{abstract}

Abstract: The way in which blind people represent themselves in the space calls for the development of certain skills to get information about their environment. One of the possibilities to get this experience could be through spatial learning. Spatial learning is characterized by the ability to learn, to perform and to update places and targets (Balakrishnan, Bousquet y Honavar, 1998). This article summarizes the thesis "Spatial representation of the environment in blind people that are hapticly stimulated by a mechatronic device, DMREl, and the classic cane" to research how does this process occur. The aim of this research is focused on the study of the spatial representation that is produced, in the environment, by the effect of technological sensitive mechanisms devices which are adapted to a human being. This is referred to identify differences in strategies of spatial representation by using mechatronic device along with classic cane. The findings obtained show differences in using the device whereas this component gets better to spatial representation as it could be proved by driving several different strategies in anticipated perception and spatial update where blind people got better results.

Key words: Spatial representation, haptic, spatial update, perceptive anticipation, spatial strategies.

Palabras clave: Representación espacial, háptica, actualización del espacio, anticipación perceptiva, estrategias espaciales.

1 Profesor Universidad Pedagógica Nacional. Este documento resume la Tesis Doctoral, desarrollada en el Programa Interinstitucional de Doctorado en Educación de la Universidad Pedagógica Nacional.

lubsan@uni.pedagogica.edu.co 


\section{Introducción}

La representación espacial se convierte en un requisito para la supervivencia del ser humano. Este mecanismo lo habilita a interactuar de manera efectiva y eficiente con su ambiente (Kamil, 1994 y Shettleworth, 1998). Tareas como cazar para alimentarse, localizar un refugio, evitar los predadores y recordar lugares peligrosos requieren un sistema confiable, en el cual los elementos importantes del mundo y sus relaciones con su cuerpo y otros elementos puedan ser representados (Healy, 1998 y Jacobs, 1995).

En relación con los invidentes, su calidad de vida depende en gran medida de su habilidad para inferir información de decisiones espaciales, dadas por medio del procesamiento y síntesis de la información espacial de una variedad de situaciones a diferentes escalas (Golledge, 1993). El sistema representado, considerado un modelo del ambiente, ha sido condicionado por la representación espacial o mapa cognitivo. El mapa cognitivo se genera con la experiencia sensorial producida por la observación que se transforma en conocimiento y comprensión del entorno (Jacobson, 1998). Para el caso de los invidentes, la falta de visión genera una descompensación de los órganos sensoriales que le permiten al individuo hacerse una representación espacial del medio que lo rodea. Esta carencia sensorial es una limitante en el procesamiento de información del entorno que se podría compensar con el uso de sistemas artificiales.

Un interrogante que surge con la utilización de estos sistemas puede formularse así: ¿proveer de una apropiada información espacial mediante canales compensatorios (Lahav y Mioduser, 2001) puede contribuir a la representación espacial de las personas invidentes? La respuesta a este interrogante nos ha llevado a estudiar la representación espacial que resulta como producto de la adaptabilidad de estos sistemas compensatorios. La evidencia de estas ayudas estaría representada en la manera en que estas personas configuran el espacio. Por tanto, el trabajo desarrollado en la investigación busca observar la disposición que una persona invidente hace del entorno y las estrategias utilizadas en la búsqueda de objetos, usando dos medios tecnológicos: un Dispositivo Mecatrónico para la Representación Espacial en Invidentes -DMREI- que se integra al sistema perceptual del invidente a través de los sensores de la piel (López, Sanabria, Sarmiento, Ibáñez y Valencia, 2005) y el bastón clásico.

El desarrollo de este documento resume los componentes de la investigación doctoral. Los primeros apartes presentan una síntesis de la literatura sobre representación, disposición y mapeo del espacio, que nos ubican en un escenario sobre el cual podemos dar razón de los antecedentes que han abordado el estudio de la representación espacial tanto en personas videntes como en personas invidentes. Este estudio permite analizar las estrategias manejadas en la exploración del entorno, la modelación y la anticipación perceptiva de las personas invidentes que intervienen en la experiencia. El problema de investigación se aborda a partir de la exploración háptica y los dispositivos tecnológicos que son utilizados como una ayuda aumentativa para que los invidentes exploren y se representen el espacio siguiendo una 
estrategia de movilidad y orientación. En esta parte del documento se hace un planteamiento de hipótesis para establecer las diferencias entre el uso del dispositivo mecatrónico, DMreI, y el bastón clásico, los cuales se consolidan en la medida en que se realiza el análisis cualitativo. La validez del análisis se incluye como el elemento que permite la modelación tanto del investigador como de los sujetos que participan en la experiencia. La parte final del documento se refiere, en primer lugar, a la metodología que se aborda desde el estudio cualitativo de casos, utilizando escenarios naturales y la descripción de técnicas de procesamiento de información verbal y gráfica que explican los procesos de razonamiento seguidos por los invidentes cuando exploran el espacio. En segundo lugar, se hace un análisis de la externalización de pensamiento del invidente en su proceso de navegación e identificación de objetos en el escenario. Este análisis utiliza las técnicas verbal y gráfica descritas en la metodología para identificar las estrategias seguidas por los sujetos en la configuración del espacio cuando utilizan los medios tecnológicos como ayuda aumentativa. En este contexto, también se realiza un análisis de la anticipación perceptual y la representación espacial en el espacio manipulatorio, que diferencian el uso de dispositivos y sirven como complemento de la investigación. La parte final del documento hace una descripción de las conclusiones obtenidas del estudio para determinar las diferencias, corrobora los objetivos y las hipótesis planteadas en esta investigación y proyecta sus resultados a la aplicación pedagógica, técnica y científica en beneficio de los discapacitados visuales.

\section{Representación espacial}

El presente trabajo hace una distinción entre la representación interna que una persona invidente construye del ambiente y la externalización de esta representación, simbolizada en términos tangibles. En este sentido, se considera la representación espacial como el razonamiento mental acerca del ambiente. El resultado se traduce en las imágenes mentales, definidas como las construcciones de la memoria de trabajo, estrechamente relacionadas con la percepción visual (Barkowsky, 2002). Estas imágenes son concebidas como modelos mentales (Johnson-Laird, 1983), los cuales incluyen información visual y espacial (Kosslyn, 1994).

Estudios sobre aspectos espaciales de los modelos mentales han generado resultados consistentes en los cuales se muestra que las personas construyen modelos mentales espaciales que contienen información acerca de relaciones y distancias espaciales entre los objetos (Rinck, Williams, Bower y Becker, 1996 y Bestgen y Dupont, 2003). La construcción de un modelo mental requiere de la integración y transformación prolongada de varias piezas de información (Zwaan y Radvansky, 1998). Asimismo, el desarrollo de un modelo mental del mundo espacial inicia con la entrada sensorial del ambiente.

Stevens y Coupe (1978), en un experimento para exhibir la estructura jerárquica de la memoria humana, preguntaron a estudiantes de la Universidad de California acerca de la orientación relativa de dos lugares con respecto a un sistema de referencia geográfico global. Las respuestas dadas llevaron a argumentar a los investigadores que los 
participantes derivaron la localización relativa de los dos sitios desde la posición relativa de dos estados en los cuales estaban localizados. A guisa de ejemplo: Bogotá está ubicada en Cundinamarca al sur de Tunja, que está localizada en Boyacá. Con la respuesta, se asume que los participantes construyeron una imagen mental de la posición relativa de dos sitios con base en alguna información disponible (Barkowsky, 2001).

Esta inferencia nos permite suponer que los modelos del ambiente construidos por personas invidentes se asimilan a la externalización de sus imágenes mentales o modelos mentales. Con esta suposición, podemos sugerir una forma de externalización, proyectada en la construcción de mapas táctiles con los cuales se realiza el estudio de la representación espacial de personas invidentes. En síntesis, el proyecto de tesis se soporta en las imágenes mentales o modelos mentales de personas ciegas que evidencian por medio de la modelación. Su relación con el aprendizaje espacial incluye la acción de algunas partes del cerebro implicadas en el procesamiento de información espacial, como el hipocampo.

\section{Disposición del espacio y mapeo cognitivo}

Las personas para desplazarse de manera independiente, deben alimentarse continuamente de información espacial. La habilidad requerida para realizar una tarea de movilidad depende en gran medida de la naturaleza de la información espacial que proviene del funcionamiento del sistema perceptual. El alcance del sistema perceptual depende del espacio observado en el entorno desde una posición y en un instante de tiempo. El alcance está determinado por el espacio inmediato, mediante el cual el movimiento es seguro y el espacio remoto en el que los mojones pueden ser detectados. Así mismo, este contexto determina la medida, referida a la anticipación perceptual y a la integración de la percepción serial de hechos espaciales adquiridos en diferentes ocasiones, las cuales se requieren para la construcción de la representación del espacio en la memoria (Foulke, 1996).

Foulke plantea que la precisión y la manera en que se realiza una tarea de movilidad dependen de la información espacial suministrada por el funcionamiento del sistema perceptual. Según él, para realizar una tarea de movilidad, las personas videntes e invidentes deben conocer las características espaciales estacionarias y el movimiento de los objetos. Este conocimiento es indispensable para predecir la posición de los objetos y actualizar la posición del individuo relativa al ambiente. Esta actualización del espacio se define como la habilidad que tienen las personas videntes e invidentes para codificar y aprender la disposición de múltiples localizaciones del espacio, relativas a un origen común y, entonces, actualizar espacialmente su posición relativa a las localizaciones mientras ellos caminan (Loomis, Klatzky, Golledge, Cicinelli, Pellegrino y Fry, 2002 y Klatzky Lederman, 2003).

Los autores antes mencionados investigan esta habilidad de las personas para actualizar mentalmente la localización de un objetivo que ha sido visto, tocado o escuchado desde un punto de observación estacionario. En otras palabras, su investigación se refiere a 
la habilidad de las personas para cambiar la representación de ellos mismos, relativa a su ambiente, de tal forma que ellos se puedan mover (Klatzky y Lederman, 2003). Para realizar la experiencia, los investigadores utilizaron entradas auditivas, lenguaje espacial descriptivo versus sonido proveniente de los objetos y visión. Los participantes aprendían la localización de objetos y luego se probaban con actualización espacial, practicando con un ejemplo visual. En la fase experimental, los sujetos videntes con los ojos vendados y los invidentes recibían estímulos auditivos verbales y sonoros que especificaban la ubicación de objetivos. Estos estímulos los convertían en imágenes espaciales que luego actualizaban mientras caminaban. Los registros tanto de distancia como de acimut muestran que los invidentes fueron capaces de actualizar la ubicación de los objetos en las diferentes condiciones experimentales con muy pocas diferencias con respecto a los videntes.

La continuidad del proceso estudiado por Loomis et, al. (2002) y Klatzky et, al. (2003), está desarrollada en la investigación que resume este documento, en la que interpreta las imágenes mentales producidas por los invidentes en la exploración de un escenario cuando reciben estímulos táctiles. Este proceso implica el estudio de la externalización de la imagen mental que se da mediante la modelación háptica y la descripción verbal como producto de la utilización de un dispositivo que sensa información del medio y la convierte en estímulos táctiles. Con esta distinción, el concepto de representación se traduce en el mapeo del ambiente, definido a partir de los mojones distribuidos espacialmen- te (Waxman, Seibert, Bernardon y Fay, 1993) con lo que se podrían determinar patrones de configuración del entorno explorado.

Según estos autores, esta aproximación es análoga al reconocimiento de objetos, lo cual lleva a explorar los detalles de un objeto desde una posición estratégica y a cuantificar estas visiones en un conjunto finito de categorías de propiedades del objeto. Esta posición se generaliza a la exploración del ambiente, dada a partir de varios puntos estratégicos, cuantificando los patrones espaciales conformados por mojones en un conjunto finito de categorías de localización. De esta forma, la combinación y reconocimiento de mojones y patrones espaciales de mojones habilitan una aproximación basada en la visión para representar los objetos y el ambiente. Por analogía, la transición de categorías de localización en el ambiente produciría un mapa cuyos parámetros espaciales podrían ser representados a nivel egocéntrico o alocéntrico (Klatzky, 1997), permitiendo proveer las coordenadas de la localización de los objetos en el ambiente.

El término "mapa cognitivo", acuñado por Tolman (1948), está referido a la representación interna que una persona hace de las características del ambiente percibido y las relaciones espaciales acerca de los objetos (Golledge, 1999). La habilidad para aprender una ruta y repasarla desde la memoria es un aspecto que corresponde al conocimiento del mapa cognitivo elaborado por las personas. Este conocimiento incluye todos los aspectos de codificación, procesamiento y recuperación de información acerca del ambiente (Blades et 
al., 2002 y Golledge, 1999). Down y Stea (1973, p. 9) definen el mapeo cognitivo como "un proceso compuesto de una serie de transformaciones psicológicas por medio de las cuales un individuo adquiere, almacena, recupera y decodifica información acerca de localizaciones relativas y atributos del fenómeno en su ambiente espacial cotidiano".

En un amplio sentido, un mapa cognitivo puede ser pensado como un modelo interno del mundo en que vivimos (Golledge y Stimpson, 1997). Existen dos elementos que se unen para conformar el mapa cognitivo: la cognición espacial y el conocimiento del mundo (Kitchin, 1994). El componente espacial concierne a la estructura, relaciones y entidades del espacio (Hart y Moore, 1973), mientras que el componente ambiental se relaciona con los atributos físicos que componen un lugar (Hart y Conn, 1991).

El mapeo cognitivo, como un proceso de razonamiento espacial, nos indica la manera en que se movilizan los invidentes y qué información se requiere en la movilidad, lo mismo que la manera en que esta información está distribuida en el ambiente. Para estudiar estos procesos, nos centramos en la exploración háptica como un medio para identificar la representación del ambiente. La exploración háptica consiste en localizar los objetos junto con sus propiedades utilizando el tacto. Esto le permite al individuo actualizar los lugares por donde va caminando. La representación mental que elabora el sujeto de la ubicación de objetos y rutas le facilita movilizarse en el entorno.

El sistema háptico de los humanos consiste en la entrada sensorial, la entra- da motora y los componentes cognitivos del sistema cerebral (Oakley, McGee, Brewster y Gray, 2000). En este sentido, la háptica se refiere a alguna cosa relacionada con el tacto. Para Fritz, Way y Barner (1996), la háptica comprende el tacto y la información cinestésica de tal forma que la definen como un sentido de posición, movimiento y fuerza.

La exploración háptica de una región delimitada de una escena sin la visión genera una representación más verídica de los límites del entorno, reduciendo los errores unidireccionales que caracterizan la extensión del límite (Gottesman e Intraub, 2003). Este proceso involucra el tacto y el movimiento. La modalidad háptica provee al perceptor con un sucesión de visiones parciales de un mundo continuo, debido a que de alguna manera el resultado de la representación mental refleja la coherencia y continuidad del espacio circundante (Intraub, 2004).

La háptica estudia e investiga la manera en que puede combinarse la modalidad sensorial del tacto con un mundo virtual. La información que se extrae mediante la háptica es intermodal, porque integra, al menos, dos modalidades perceptivas: la propiocepción, que relaciona toda la información sensorial acerca del estado del cuerpo (Oakley et al., 2000). Esta modalidad informa mediante información cinestésica del estado del aparato esquelético-locomotor (tono muscular, posición del cuerpo, equilibrio, etc.). Y la dermocepción o tacto, que contribuye aportando información sobre la textura y la presión mecánica sobre la piel. La información cutánea se refiere a la sensación que surge de la piel cuando entra en contacto con un objeto, como es el caso de los actuadores vibro- 
táctiles del dispositivo utilizado para la experiencia con los invidentes.

\section{Estrategias en la configuración del espacio}

La formación de representaciones mentales de estructura espacial en grandes y pequeños espacios realizada por personas invidentes está mediada por el empleo de estrategias. En este proceso, el manejo de estrategias facilita la codificación de relaciones espaciales y la estructuración de objetos en el ambiente (Ungar, Blades, Spencer y Morsley, 2004).

En relación con el significado psicológico del término "estrategia", dichos autores distinguen dos sentidos para esta expresión: el primer sentido se refiere a las estrategias de codificación que se relacionan con la manera en que la información espacial es codificada en la memoria. Este proceso tiene que ver con los tipos de señales utilizados, casos en los cuales las localizaciones se codifican respecto al cuerpo del individuo, relativas a la localización de una señal simple o en relación con un marco de referencia espacial externo (Millar, 1994). Como un ejemplo de este tipo de estrategias, tenemos las estrategias espaciales egocéntricas y alocéntricas. Millar considera que la tendencia de los invidentes es utilizar estrategias egocéntricas debido a que son altamente eficientes en la realización de tareas espaciales. Para Millar, las estrategias son consideradas como "formas opcionales de codificación" en la medida en que éstas son intercambiables.

O'Keefe y Nadel (1978) sugieren la existencia de dos tipos de estrategias: estrategias espaciales egocéntricas y estrategias espaciales alocéntricas. Las estrategias espaciales egocéntricas, centradas en el cuerpo, se refieren a la discriminación de un lugar geométrico en el espacio con referencia a la línea media del cuerpo, al meridiano visual vertical o al movimiento relativo a sí mismo. La estrategia egocéntrica podría utilizar información visual, pero en ausencia de este tipo de información, como es el caso de los invidentes, se podría usar información auditiva, táctil u olfativa. Este sistema de procesamiento compromete la formación del hipocampo y el córtex parietal (Aguirre, Zarahn y D'Esposito, 1998) y podría incluir el giro temporal inferior, el giro fusiforme y el giro frontal medio que mostrarían una conexión con la formación bilateral del hipocampo y con el giro hipocampal.

Las estrategias espaciales alocéntricas están centradas en el ambiente. Los seres humanos, para su desplazamiento, requieren activar procesos alocéntricos y egocéntricos, con lo cual adquieren un mejor conocimiento espacial de la relación entre la persona y el objeto que se altera en la medida que cambia el movimiento (egocéntrico) y una relación más estable entre un objeto y otro, en la que la estrategia alocéntrica fija su mapa cognitivo (Sholl, 1996). El desarrollo del constructo alocéntrico podría involucrar las mismas áreas utilizadas en la estrategia egocéntrica. Shelton y Gabrieli (2002) identifican algunas áreas, como el giro fusiforme, el giro temporal inferior y el córtex parietal inferior -que podrían incrementar la conexión con la formación del hipocampo y el giro hipocampal- junto con el giro lingual. La activación del giro lingual puntualiza la función lingüística en la represen- 
tación espacial (Aguirre et al., 1998 y King, Burgués, Hartley, Vargha-Khadem y O’Keefe, 2002).

El segundo sentido se refiere a la secuencia de comportamientos que manifiesta una persona cuando explora la disposición espacial de su entorno, así como a las decisiones que toma y otras actividades metacognitivas de estos comportamientos. Los autores se refieren a este tipo de estrategias como estrategias comportamentales. El enfoque en este tipo de estrategias permite inferir diferencias en los modelos representados por los invidentes.

La identificación de patrones se vincula a las estrategias manejadas en el reconocimiento de lugares y configuración del ambiente. La tarea de identificar patrones es un objetivo trazado en el trabajo de investigación y lleva a establecer formas del área explorada por los invidentes. Una persona ciega, cuando navega en el espacio, podría inferir o imaginar zonas delimitadas por mojones. Estos parámetros de orientación lo llevarían a recordar y representar la disposición del escenario explorado. El estudio que sigue es un acercamiento a la intención de poder identificar patrones de configuración del ambiente, con los cuales se podría comprender la representación espacial de personas invidentes.

Hill et, al. (1993) investigaron acerca de la representación mental del espacio. Su experimento consistió en inducir a personas invidentes a explorar libremente un espacio abierto limitado con el fin de localizar cuatro objetos y recordar su posición. El proceso fue grabado en video. Los patrones espaciales encontrados en la grabación fueron codificados en diferentes estrategias que el autor clasificó. Hill condujo a los participantes a hacer una serie de juicios de dirección entre los sitios de ubicación de los objetos y la precisión de su respuesta recordada. Este proceso lo realizó con el objeto de investigar la precisión de las respuestas de dirección en relación con el comportamiento exploratorio usado por los participantes. Para lograr su propósito, Hill clasificó a los participantes en términos de su realización y comparó las estrategias empleadas por los mejores realizadores contrastados con los peores. El autor encontró que los mejores realizadores tendían a emplear una amplia variedad de estrategias. Asimismo, ellos utilizaban estrategias que facilitaban el desarrollo de relaciones alocéntricas.

Gaunet, Martínez y Thinus-Blanc (1997), en una investigación para determinar las estrategias utilizadas en tareas espaciales táctiles, condujeron a adultos ciegos congénitos y tardíos y a personas videntes en la tarea de explorar durante un minuto la disposición de cinco formas geométricas, ubicadas en una tabla cuadrada de un metro. Los patrones resultantes de la disposición explorada son grabados en video. Una vez que los participantes exploran la escena, el experimentador altera la disposición de los objetos. Los participantes, entonces, identifican el nuevo escenario con base en tres condiciones: en relación con ellos mismos y el escenario, moviéndose 180 grados alrededor de la tabla y rotando la tabla 180 grados. Los autores, basados en la secuencia de visitas que realizaban los sujetos a las formas geométricas, pudieron identificar en las grabaciones dos tipos de patrones 
exploratorios: una exploración cíclica, que incluye visitas a diferentes figuras, y una exploración adelante y atrás, que incluye el movimiento repetido entre dos figuras. Los autores encuentran que una alta proporción de patrones adelante y atrás se asocian a una mejor realización. De la misma manera, encuentran que los invidentes congénitos participantes en la experiencia son los que más se incluyen en patrones cíclicos.

\section{La representación espacial de invidentes como el problema de investigación}

La representación espacial con estimulación táctil se asume como objeto de estudio de la investigación teórica. En esta dimensión, se hace necesario comprender, en primera instancia, el sistema háptico como mecanismo de procesamiento de información; en segunda instancia, las posibilidades de representación con la utilización de dispositivos tecnológicos como sistemas de extensión de los órganos sensoriales y, en tercera, la representación con mapas táctiles en lo que se refiere a recuperación de la memoria espacial.

El proyecto que se desarrolló forma parte de la investigación en discapacidad visual. El campo de aplicación se relaciona con la movilidad y orientación de personas invidentes. A partir de la comprensión de estos temas, el problema de investigación se centra específicamente en estudiar la externalización de la representación espacial del ambiente con estimulación táctil de dos sistemas tecnológicos: el bastón clásico y un dispositivo mecatronico, DMREI, que traduce señales visuales en señales hápticas. Este dispositivo fue desarrollado de manera experimental en una investigación previa (López et al., 2005).

El proceso de investigación recurre a la disposición y modelación del ambiente como mecanismos de representación espacial en discapacitados visuales. Con esto se cuestionan las formas de configuración que el invidente se hace del ambiente, cuando recibe información de dos medios a través de señales que estimulan algunos de sus órganos sensoriales no visuales. Para dar sentido a esta deliberación, es necesario indagar, en primera instancia, acerca de las diferencias de las representaciones espaciales a partir de las estrategias utilizadas en la exploración táctil, una vez que el invidente ha explorado el ambiente, primero con el bastón y luego con el dispositivo; en segundo lugar, acerca de la riqueza de la representación a partir de la configuración y la variedad de información recuperada del entorno que ha sido explorado previamente por los invidentes, utilizando los dos medios de estimulación táctil.

En relación con el problema de esta investigación, se plantean los criterios fundamentales para alcanzar el rigor de validez del método investigativo al que apunta esta propuesta. Por un lado, la perspectiva de la investigación se dirige a la diferenciación de la representación espacial y, por otro, la validez se enmarca en las condiciones de la experiencia: la reproducción de mapas con la mayor fidelidad y su comparación con el análisis verbal.

Con esta investigación se espera que los invidentes, con el apoyo del dispositivo mecatrónico y el bastón, generen diferencias que permitan establecer nuevos modelos de entrenamiento apoyados en estos recursos tecnológicos. Por tanto, 
el logro de los objetivos planteados en el ámbito investigativo es una meta que permitirá identificar estas diferencias.

Con base en el análisis de estrategias y la comparación de modelos táctiles, se busca establecer un esquema de diferenciación de los dos elementos en prueba que nos permita formular un avance en la representación del espacio con el apoyo de estrategias más efectivas para los procesos de aprendizaje, en movilidad y orientación, orientados por los docentes.

La reducción de las expresiones verbales a estructuras formales y la abstracción de modelos permiten la comparación que le da validez al estudio de la representación espacial. Esta investigación desarrolla un modelo de comparación entre la verbalización y la exploración física de una escena. La validez se da en la confrontación de la reproducción de una escena desde acciones reales de la videograbación con los datos verbales que manifiesta la persona invidente. Esta comparación combina tres situaciones: la descripción verbal de la escena por el invidente, la reproducción fiel del recorrido en mapas gráficos con señalamiento de puntos de encuentro por parte del investigador y la reconstrucción táctil del invidente. Las tres situaciones validan las estrategias generadas a partir del análisis verbal y las estrategias generadas a partir de los modelos obtenidos gráficamente.

Las estrategias inferidas a partir de las estructuras derivadas de las expresiones verbales y su correspondencia con las estrategias inferidas a partir de los mapas reproducidos en la observación de la grabación en video, tanto en la locomoción como en la exploración háptica, permitirán identificar las diferencias en la representación espacial manejadas por el invidente. En esta comparación, la reproducción de la grabación le da validez a las estructuras obtenidas del análisis verbal.

\section{Metodología y análisis comparativo de los procesos de representación}

\section{Proceso metodológico}

La investigación que se desarrolla en esta tesis se enfoca en el estudio cualitativo de casos (Montero y León, 2002 y Anyon, 1981). Para ello, se ha decidido utilizar como estrategia metodológica el análisis cualitativo de reportes verbales, siguiendo la guía práctica de Chi (1997) sobre análisis cualitativo de datos verbales y la obra de Ericson y Simon (1993) sobre análisis de protocolos y el análisis gráfico de los mapas reproducidos en la exploración. El empleo de estas técnicas se soporta en los estudios realizados por Ungar, Blades y Spencer (1997), quienes las utilizaron para identificar diferencias individuales en el uso de estrategias para explorar y organizar información, después de que los invidentes aprendían un mapa complejo de una ciudad, para luego reconstruirlo desde su memoria. Su aplicación sistemática nos permite, por un lado, ver las estrategias seguidas por cada uno de los sujetos que participan en el estudio y, por otro, comparar las representaciones imaginadas por estas personas cuando recuerdan el entorno explorado. El proceso incluye la descripción verbal, explícita en "informes verbales recurrentes", en los cuales el individuo habla en voz alta a medida que realiza alguna operación de búsqueda de objetos (Maldonado, 2001), y la modelación de la configuración del escenario, en la que el sujeto reconstru- 
ye la disposición de los objetos en un modelo a escala, mientras va recordando su ubicación (Ochaíta y Huertas, 1993).

El grupo de sujetos que participan en el proceso investigativo lo conforman cuatro personas invidentes congénitas del Centro de Rehabilitación de Personas Ciegas, Crac, cuyas edades oscilan entre quince y veinte años. La escogencia de este número de personas se justifica por el tipo de análisis que se plantea (análisis cualitativo de reportes verbales). Algunos estudios han utilizado grupos pequeños de personas, como el propuesto en esta investigación (Jacobson, 1998, Gladstone, 1991 y Wang y Spelke, 2000. Las personas ciegas han desarrollado habilidades en cuanto a orientación y movilidad con manejo del bastón, han adquirido habilidad para comunicarse con iguales y videntes, han recibido entrenamiento en el manejo de mapas táctiles y tienen cierta independencia para realizar actividades de la vida diaria.

El proceso investigativo incluye el estudio de casos individuales, en el cual cada persona utiliza dos elementos tecnológicos para explorar el entorno: el dispositivo mecatrónico -DMREI- y el bastón. El aprendizaje de los individuos que son objeto de estudio está dado, por un lado, en función del manejo del dispositivo táctil -DMREI- utilizado para la experiencia y, por otro, en función del reconocimiento de figuras geométricas y la exploración de escenas en el ambiente natural y en el mapa táctil. La exploración del escenario natural se da con los dos elementos tecnológicos.

Cada uno de los sujetos que forma parte de la experiencia se enfrenta al desafío de buscar cinco objetos (formas geométricas) dispuestos en el área cua- drada de un salón limitado por cuatro paredes. Esta distribución coincide con los estudios realizados por Loomis et al. (1993) y Rieser, Guth y Hill, (1986) con respecto al número de objetos y su distribución. Los sujetos primero reciben instrucciones de decir en voz alta lo que están pensando a medida que van recorriendo el escenario y localizando los objetos. Una vez que los sujetos han atendido a las observaciones, inician de manera libre la exploración del escenario por el centro de uno de los extremos del área demarcada. A medida que cada individuo se va movilizando, debe indicar de manera verbal la dirección que sigue cuando encuentra un objeto hasta alcanzar el último. Cada vez que encuentra un objeto, se indaga acerca de la posición en relación con los otros. Cuando los sujetos han terminado de explorar el escenario, se inducen a caminar por los sitios donde ellos creen que estaban los objetos para que indiquen su disposición en el escenario. Los dos recorridos son grabados en video con el fin de reconstruir de la forma más fidedigna el mapa de navegación y localización de objetivos para el análisis posterior. El proceso final consiste en suministrarle un tablero que representa el escenario a escala y un conjunto de objetos a escala para que el sujeto construya un modelo de la escena que acaba de explorar, recordando la disposición de los objetos. Todo el proceso es realizado primero con el bastón y luego con el dispositivo -DMREI-. Las acciones del sujeto tanto en el escenario natural como en el mapa táctil, así como sus expresiones verbales, son grabadas utilizando una cámara de video.

Para complementar el estudio, se le presenta al sujeto nuevamente el tablero 
con la ubicación correcta de los cinco objetos, con el fin de que el individuo explore de manera táctil la ubicación de los mismos. Esta distribución sigue los estudios de Gaunet, Martínez y ThinusBlanc (1997) y Ungar, Blades y Spencer (1997), quienes realizan experiencias con invidentes en la exploración de la disposición de cinco objetos dispuestos en el área de un tablero. Una vez que el sujeto ha explorado la disposición de los objetos, se procede a reemplazar el tablero por otro vacío para que el individuo reconstruya la escena que ha logrado inferir.

\section{Análisis de la representación espacial}

\section{Anticipación perceptual}

La descripción del esquema de codificación consiste en el cálculo de las distancias desde la posición del sujeto hasta la posición de los objetos percibidos. Este patrón coincide con el alcance del sistema perceptual planteado por Foulke (1996). La operación se realiza tomando como referencia el punto donde el sujeto expresa que ha percibido un objeto. Los puntos están señalados en el mapa que ha sido reproducido de la grabación (Figura 1) en el cual se muestra la trayectoria que sigue el sujeto para ubicar un objeto, utilizando el dispositivo, -DMREILas líneas delgadas indican las distancias a cada uno de los objetos percibidos desde la posición del sujeto (posición egocéntrica). Con el análisis de estos datos, se infiere el comportamiento a nivel de anticipación perceptual antes de lograr localizar un objeto. Este proceso se repite para la búsqueda de todos los objetos, como se señala en el mapa de la Figura 2, en el cual aparece todo el recorrido que hace el sujeto durante la exploración del ambiente.

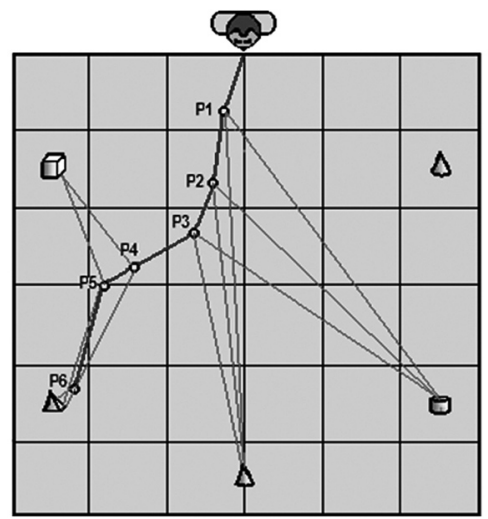

Figura 1: Mapa de la búsqueda de un objeto con indicación de las distancias.

Las distancias y la dirección tomadas en la percepción de todos los objetos se reportan en la Tabla 1 y corresponden al recorrido de un sujeto (Figura 2). A partir del análisis de las cláusulas obtenidas del esquema de codificación en el protocolo, explícitas en los datos de la Tabla 1, se infiere la estrategia que está siguiendo el sujeto en la búsqueda de los objetos, siguiendo la clasificación de Gaunet y Thinus-Blanc (1997) y Hill et al. (1993).

Para analizar la medición de la anticipación sensorial, se ha decidido tomar los últimos datos sombreados de la Tabla 1, correspondientes al seguimiento de cada objeto encontrado, siguiendo la teoría de Posner y Di Girolamo (1998), acerca de la selección de información para ser procesada por la atención, y la teoría de Scott (2000), relacionada con las entradas sensoriales que son atendidas en función de reducir la carga perceptiva. Esta realización considera un 
análisis fino de la atención que incluye el proceso seguido por el invidente a partir del momento en que esta persona percibe un estímulo que lo conduce a centrar su atención en un solo objeto hasta que logra encontrarlo. Este análisis permite hacer una clasificación de la atención en niveles: alto, medio y bajo. Para determinar los niveles de atención, se han tomado el número de puntos de percepción que orientan al sujeto al objetivo, es decir, los eventos que centran el hallazgo del objeto. Los puntos de percepción están relacionados con el alcance de la percepción y son los lugares donde el sujeto percibe un estímulo del dispositivo, DMREI. Estos puntos se encuentran señalados en la Tabla 1 y se identifican con las letras P1, P2, P3,...Pn. Tomando como referencia esta distribución, se hace una clasificación de los puntos de percepción para determinar regularidades que indican el nivel de atención.

Los datos no sombreados que anteceden este proceso, aunque no son tenidos en cuenta para calcular el nivel de atención, se consideran útiles en la identificación de las estrategias que sigue el usuario en la navegación del entorno. El proceso de comparación entre los datos y las cláusulas originadas de la verbalización es una fase del análisis que le da validez al protocolo.

La Figura 2 muestra la evolución de la atención de un sujeto cuando se centra en la búsqueda de cada uno de los objetos. En la gráfica, los números internos indican la cantidad de objetos percibidos en cada punto de percepción y cada curva indica el acercamiento o alejamiento al objeto que se está localizando.

\begin{tabular}{|c|c|c|c|c|c|c|}
\hline \multirow{2}{*}{$\begin{array}{l}\text { Punto de } \\
\text { percepción }\end{array}$} & \multirow[b]{2}{*}{ Dirección } & \multicolumn{2}{|c|}{ Distancia (m) } & \multirow{2}{*}{$\begin{array}{l}\text { Objeto } \\
\text { uno }\end{array}$} & \multirow{2}{*}{$\begin{array}{l}\text { Objeto } \\
\text { dos }\end{array}$} & \multirow[b]{2}{*}{ Estrategia inferida } \\
\hline & & $\begin{array}{c}\text { Objeto } \\
1\end{array}$ & Objeto 2 & & & \\
\hline $\mathrm{P} 1$ & $\downarrow$ & 3,8 & 4,2 & Cono & Cilindro & \multirow{6}{*}{$\begin{array}{l}\text { Tendencia del sujeto } \\
\text { a reducir el espacio } \\
\text { de búsqueda a partir } \\
\text { de la reducción de las } \\
\text { distancias al objeto y a } \\
\text { seguir una trayectoria } \\
\text { en una sola dirección } \\
\text { (frontal). El cambio de } \\
\text { dirección se da cuando } \\
\text { pierde la señal. }\end{array}$} \\
\hline $\mathrm{P} 2$ & $\leftarrow \downarrow$ & 2,9 & 3,7 & Cono & Cilindro & \\
\hline P3 & $\downarrow$ & 2,5 & 3,4 & Cono & Cilindro & \\
\hline P4 & $\leftarrow$ & 1,2 & 1,4 & Cubo & Pirámide & \\
\hline P5 & $\downarrow$ & 1,2 & 1,2 & Cubo & Pirámide & \\
\hline $\mathrm{e}$ & $\downarrow$ & -- & 0,4 & --- & Pirámide & \\
\hline \multicolumn{2}{|c|}{ Nivel de atención } & & & \multicolumn{2}{|l|}{ Medio } & $\begin{array}{l}\text { Los datos de distancia en } \\
\text { la anticipación indican } \\
\text { una aproximación } \\
\text { sistemática con cambio } \\
\text { de trayectoria para } \\
\text { orientar la búsqueda } \\
\text { hacia un objetivo. }\end{array}$ \\
\hline
\end{tabular}

Tabla 1: Caracterización de la percepción de un objeto y estrategias durante el aprendizaje del entorno de un sujeto. 


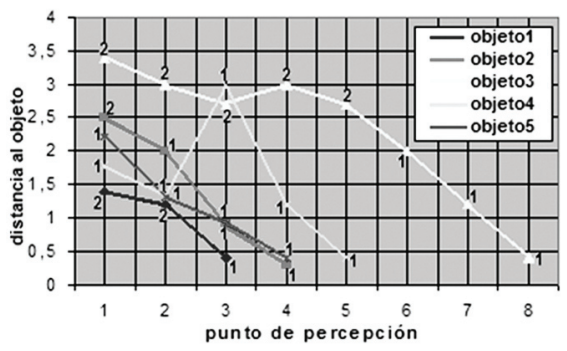

Figura 2: Evolución de la atención en el proceso de anticipación perceptiva (sujeto 1).

Una síntesis del análisis de la anticipación perceptiva a partir de los niveles de atención de las cuatro personas invidentes que participaron en la experiencia genera las siguientes inferencias:

1. El afinamiento del nivel de atención se da a partir del momento mismo en que el sujeto adquiere alguna experiencia en el manejo del dispositivo, o sea, cuando el sujeto se adapta al mismo. Este proceso de adaptación coincide con la integración del movimiento del cuerpo con los eventos perceptuales que siguen esta acción y que provienen del dispositivo, lo cual se suma al planteamiento de Hommel (2005), en lo que respecta a la planeación y control de la acción. Este proceso le permite ejercer un control en el manejo de la atención.

2. En la medida que el sujeto avanza en la búsqueda, existe un mayor afinamiento y la anticipación perceptual aumenta con la distancia al objetivo.

3. Se evidencian dos procesos de atención; uno, cuando el sujeto percibe dos objetos y otro, cuando el sujeto percibe un objeto. Una explicación para el primer proceso haría suponer un primer nivel de preparación; y en el segundo proceso, se daría una selección de la información que se procesa con la atención. Esto coincide con la contigüidad y contingencia planteadas por Hommel (2005), en las que inicialmente se asume una integración de eventos perceptuales para luego excluir los eventos sobrantes y de esta forma centrar la atención en un solo objetivo, lo cual se logra en la medida que el sujeto va adquiriendo experiencia.

4. Cuando quedan menos objetos en el escenario, caso de los dos últimos episodios, así como cuando un sujeto centra su atención en un solo objeto, se considera que existe un afinamiento en el nivel de atención. Este resultado podría tener una explicación: en tanto aumenta el nivel de complejidad (mayor número de obstáculos), existe un mayor esfuerzo cognitivo y la atención aumenta, pero es más dispersa. Cuando se reduce el número de obstáculos, existe un menor esfuerzo cognitivo y por lo cual, aunque el nivel de atención se incremente, se puede afirmar que se da un afinamiento de la atención. Esta inferencia supondría un mayor procesamiento cognitivo, cuando se exploran ambientes complejos y, en razón a este esfuerzo, el nivel de atención se haría más disperso.

5. El análisis de todos los sujetos muestra una exploración inicial al azar que luego se mejora con el incremento en el nivel de atención, lo cual podría sugerir la generación de tres etapas en el proceso 
de anticipación perceptual con el dispositivo: una primera etapa de adaptación al dispositivo, una segunda etapa de regulación de la atención y una tercera etapa de afinación, que conlleva a un acercamiento progresivo al reconocimiento de los objetos.

6. El uso del dispositivo, DMrEI, genera una afinación e incremento de la atención, facilitando la localización de los objetos. Este resultado es un indicador del potencial de este aparato para canalizar los estímulos que le permiten al invidente seleccionar la información, facilitar su procesamiento a nivel cognitivo y reducir su esfuerzo mental, como lo plantean las investigaciones de Sarmiento (2004) y López et al. (2005).

\section{Actualización del espacio}

En la actualización del espacio, se toma como referencia la descripción del ambiente que va realizando el invidente a medida que encuentra nuevos objetos. Cada vez que el sujeto encuentra un nuevo objeto, se le solicita la descripción de la localización tanto del nuevo objeto como de los otros que ha encontrado previamente. La actualización del espacio que se logra con esta descripción permite llevar un registro de la disposición de los objetos, lo cual revela el mapa que va construyendo el sujeto mientras explora el ambiente.

La Figura 3 muestra la representación que hace un sujeto en el proceso de actualización cada vez que encuentra un objeto. Esta gráfica ha sido reproducida a partir de la grabación en video y confronta la verbalización del sujeto expuesta en cada segmento con la imagen de las acciones del sujeto, tomadas del video. Los vectores representan la dirección que señala el sujeto de los objetos, la indicación del punto en el que el sujeto recibe el estímulo en la matriz háptica y la trayectoria y posición del sujeto en el momento que encuentra el objeto y verbaliza la disposición del mismo.

Al hacer un análisis de la representación de la actualización de este sujeto, mostrada en el plano de la Figura 3, se pueden identificar dos situaciones: una es que el individuo actualiza su posición en el espacio, recuperando de su memoria la posición de los objetos que ha encontrado previamente (Loomis et al., 2002 y Klatzky et al., 2003); esta recuperación le permite orientarse en el entorno. La segunda es que el sujeto maneja dos esquemas de codificación: el primero, se refiere a la codificación de la disposición del entorno en la cual indica la dirección de la ubicación de los objetos que va encontrando; y el segundo se relaciona con la codificación de los estímulos que recibe del dispositivo-DMREIque le permiten recordar su aprendizaje en el manejo del aparato.

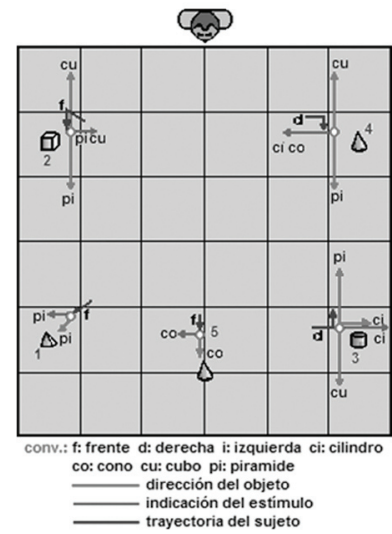

Figura 3. Actualización del espacio en la búsqueda de objetos.

Comparando las representaciones con el uso del bastón, se pudo observar 
que los sujetos reconstruyen la disposición de los últimos objetos encontrados. El bastón los limita a encontrar los objetos únicamente cuando los tocan. No existe un manejo de la anticipación perceptual que les permita orientarse en el desplazamiento. Las pocas relaciones que se establecen entre objetos, se dan con un conocimiento previo de mojones que les sirven como puntos de referencia. La actualización del espacio se da en función de los últimos objetos que el sujeto recuerda. Este hecho evidencia una representación egocéntrica.

En síntesis, se observa que los sujetos actualizan la representación del espacio en cualquier posición de su cuerpo y, además, el uso del dispositivo-DMreIles permite establecer relaciones entre objetos. Este hecho facilita el manejo de una estrategia alocéntrica (Kaztkly,
1997), con la cual ellos podrían realizar una representación configuracional del espacio explorado.

\section{Disposición del espacio}

Esta fase de análisis deja ver la representación de la disposición que hace el sujeto del ambiente en dos etapas: una es la representación que se abstrae de la verbalización del sujeto durante el aprendizaje; y la otra se relaciona con la precisión en la indicación que hace el sujeto en el ambiente y la elaboración de un modelo a escala de la disposición del ambiente después de que ha sucedido el aprendizaje. Esta última fase se refiere a la externalización del mapa cognitivo del sujeto, tal como lo experimentó Jacobson (1998) en uno de sus estudios.

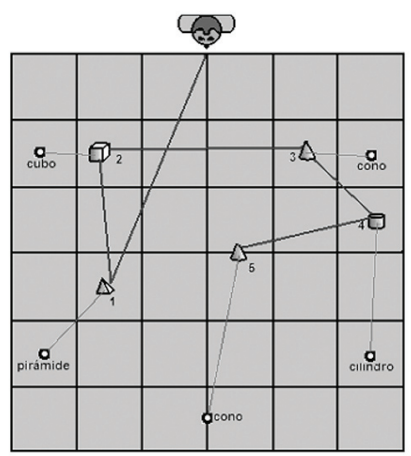

Sujeto1

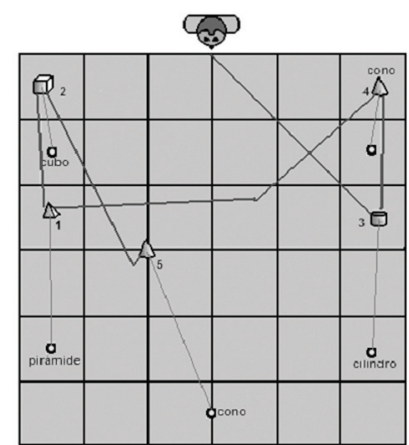

Sujeto1

Figura 4: Modelos de la disposición del espacio. 
Analizando los dos mapas de un sujeto como se ve en la Figura 4, se evidencia una disposición correcta de los objetos, lo que confirma la disposición espacial que hace el invidente en el escenario de manera verbal. Este resultado es un predictor de la capacidad del invidente para representarse el espacio de manera configuracional (Roche, Mangaoang, Commins y O'Mara, 2005). En cuanto a la precisión en la representación, donde los puntos amarillos representan la disposición de objetos antes de la exploración y los objetos representan la disposición que el sujeto realiza una vez ha terminado la exploración, se pueden observar errores en el cálculo de distancias, lo cual evidencia que esta persona no maneja una métrica precisa en la exploración del espacio. Si se comparan los dos mapas, se puede observar que el sujeto hace un ajuste en la posición de los objetos cuando realiza el modelo. Probablemente, esto se deba al manejo de una mayor precisión en el espacio peripersonal, como lo experimentaron Klatzky y Lederman (2003), y a los límites del entorno que le sirven como parámetros para lograr una mejor ubicación de los objetos en el mapa. Este factor se ubica en la extensión del límite planteado por Intraub (2004).

Los resultados obtenidos, comparando el uso del bastón con el dispositivo -DMREI- coinciden con los resultados de Jacobson (1998), en el sentido de que existe mayor eficiencia en cuanto a precisión para el manejo de la configuración del espacio con el uso de dispositivos a diferencia de la utilización del bastón. Probablemente, esto se deba a que existe mayor información con la anticipación perceptiva que genera el dispositivo, lo cual genera una representación del espacio más precisa.

Los modelos obtenidos de la disposición de objetos en el ambiente de los sujetos hacen inferir que las personas invidentes son capaces de hacer una buena representación del espacio con el uso de dispositivos tecnológicos. Esto permite un acercamiento a la representación configuracional del ambiente (Roche et al., 2005). Aunque no son muy precisos en el manejo de distancias, la interacción con mapas táctiles para la modelación del espacio, al parecer, ayuda a precisar el manejo de distancias y la dirección de localización de los objetivos, tal como lo demostraron Espinosa, Ungar, Ochaíta, Blades y Spencer (1998) y Ungar et al. (1994) en sus investigaciones con el uso de mapas táctiles.

\section{Conclusiones}

El manejo de la anticipación perceptiva es importante en la configuración del espacio. Esto difiere en los dos dispositivos: mientras que el -DMrEI-ayuda a la anticipación perceptual y la configuración del espacio es mejor, con el bastón existe una anticipación perceptiva muy baja que depende del recuerdo de mojones y, por tanto, se prevé que existe una representación espacial menos eficiente. Probablemente, cuando el sujeto tiene una anticipación perceptiva, existe un mejor procesamiento de información que genera un mayor aprendizaje, es decir, existe una mejor compilación del conocimiento del entorno. Similar a como lo plantean Loomis et al. (2002) y Klatzky et al. (2003), las imágenes espaciales formadas con el uso del bastón son más frágiles y requieren mayor procesamiento de información 
para actualizarse. Esta demanda podría influir en el mejoramiento de la representación espacial del sujeto, lo cual produciría un sesgo en la disposición de los objetos.

Se infiere que el uso del dispositivo ayuda a organizar la manera en que el sujeto configura el ambiente. Esto se podría visualizar en los estímulos emitidos por el aparato que le indican la presencia de mojones en el entorno. Al parecer, el sujeto tiende a memorizar estos estímulos de tal forma que, al recordarlos, se puede hacer una mejor configuración del espacio, como quedó demostrado en los mapas.

El manejo de estrategias es un factor importante que diferencia la utilización del dispositivo respecto de la utilización del bastón. Los resultados de las investigaciones de Gaunet, Martínez y Thinus-Blanc (1997) y Hill et al. (1993) demuestran que el manejo de una variedad de estrategias consolida una mejor representación del espacio. Basados en estas conclusiones, y de acuerdo con el análisis de los mapas de navegación y los reportes verbales, se evidencia que el uso del dispositivo generó el manejo de una mayor variedad de estrategias.

Esta variedad incluía el manejo de estrategias de rejilla, adelante-atrás, perímetro a objeto, etc. En la utilización del bastón, predominó el manejo de estrategias cíclicas y de perímetro. Esto confirma la tesis de Gaunet et al. y de Hill et al., según la cual las mejores realizaciones se logran con el uso del dispositivo.

Con base en la clasificación de O'Keefe y Nadel (1978) y Klaztky (1997), respecto al manejo de estrategias alocéntricas y egocéntricas, se puede visualizar que, a pesar de que los invidentes son egocéntricos, como lo afirma Millar (1994), el manejo del dispositivo por parte de los sujetos genera una mayor posibilidad para establecer relaciones entre los objetos. Esta característica forma parte de una estrategia alocéntrica. Al parecer, el uso del dispositivo ayuda a consolidar el manejo de estrategias alocéntricas.

Los estímulos que el invidente recibe del dispositivo son más variados, por tanto, existe un mayor procesamiento de información, lo que implica una mejor codificación del espacio explorado. Esto se contrasta con una disposición de los objetos más precisa cuando se utiliza este medio, tal como quedó demostrado en la reconstrucción de los mapas de los invidentes.

Respecto a la actualización del espacio planteada por Loomis et al. (2002) y Klaztky et al. (2003), el uso del dispositivo, DMREI, le permite al invidente una localización correcta de los objetos. Esto se suma a la cantidad de objetos reconocidos en cada punto de actualización.

Considerando finalmente el punto de vista pedagógico, se concluye que el entrenamiento de atención, como lo demuestra la investigación, sería un factor que debe tenerse en cuenta para que el invidente actúe con cierto grado de anticipación perceptiva. De la misma forma, el entrenamiento con el uso de mapas táctiles ayudaría a la identificación de lugares en contextos reales.

Otro aspecto por tener en cuenta se relaciona con la posibilidad de diseñar dispositivos que le permitan al invidente actuar con una mayor base de conocimiento, producto de la información que reciben a través de la estimulación táctil. Esta propiedad quedó demostra- 
da con el uso del dispositivo-DMREI-con el cual existe un mayor número de señales que transmiten información a la persona invidente que le ayudan a localizar objetivos y, por consiguiente, a hacerse una buena representación del espacio. Respecto al manejo de estrate-

\section{Referencias bibliográficas}

Aguirre, G., Zarahn, E. y D'Esposito, M. (1998). An area within human ventral cortex sensitive to "Building" stimuli: evidence and implications. Neuron, 21, 373-383.

Anyon, J. (1981). Social class and school knowledge. Curriculum Inquiry, XI, 3-42.

Balakrishnan, K., Bousquet, O. y Honavar, V. (1998). Spatial learning and localization in rodents: a computational model of the hippocampus and its implications for mobile robots. Adaptive Behavior. En preparación.

Barkowsky, T. (2001). Mental processing of geographic knowledge. En D. R. Montello (ed.) (s. f.), Spatial information theory-foundations of geographic information science (pp. 371-386). Berlín: Springer.

Barkowsky, T. (2002). Mental representation and processing of geographic knowledge. A computational approach. Berlín: Springer.

Bestgen, Y. y Dupont, V. (2003). The construction of spatial situation models during reading. Psychological Research, 67, 209-218.

Blades, M. (1990). The reliability of date collected from sketch maps. Journal of Environmental Psychology, 10, 327-340. gias, esta investigación deja abierta la posibilidad de adaptar algunas para el aprendizaje en orientación y movilidad de las personas invidentes. Se considera que el entrenamiento con la utilización de estas estrategias podría mejorar las condiciones de desplazamiento de estas personas.

Chi, M. (1997). Quantifying qualitative analyses of verbal data: a practical guide. The journal of the Learning Sciences, 6(3), 271-315.

Downs, R. y Stea, D. (1973). Cognitive maps and spatial behavior: process and products. Image and Environments Chicago: Aldine Publishing Company; pp. 8-26.

Ericsson, K. y Simon, H. (1993). Protocol analysis: verbal reports as data. Cambridge: MIT Press.

Espinosa, M., Ungar, S., Ochaíta, E., Blades, M. y Spencer, C. (1998). Comparing methods for introducing blind and visually impaired people to unfamiliar urban environments. Journal of Environmental Psychology, 18, 277-287.

Foulke, E. (1996). The roles of perception and cognition in controlling the mobility task. Documento del International Symposium on Orientation and Mobility. Trondheim, Noruega.

Fritz, J. , Way, T. y Barner, K. (1996). Haptic representation of scientific data for visually impaired or blind people. Technology and People With Disabilities Conference.

Garling, T. y Golledge, R. (2000). Cognitive mapping and spatial decisión-making. En R. Kitchin y S. Freundschuh (eds.) 
(s. f.), Cognitive mapping: past, present and future. Londres: Routledge.

Gaunet, F., Martínez, J. y Thinus-Blanc, C. (1997). Early-blind subjects' spatial representation of manipulatory space: exploratory strategies and reaction to change. Perception, 26, 345-366.

Gladstone, M. (1991). Spatial cognition and mapping abilities: a comparison of sighted and congenitally bind individuals. Disertación no publicada, Universidad de Edimburgo.

Golledge, R. (1993). Geography and the disabled: a survey with special reference to vision impaired and blind populations. Transactions of the institute of British Geographers, 18, 63-85.

Golledge, R. (1999). Human wayfinding and cognitive maps. En R. Golledge (ed.), Wayfinding behaviour (pp. 5-45). Baltimore: Johns Hopkins University Press.

Golledge, R. y Stimpson, R. (1997). Spatial behaviour: a geographic perspective. Nueva York: The Guilford Press.

Gottesman, C. e Intraub, H. (2003). Constraints on spatial extrapolation in the mental representation of scenes: viewboundaries versus object-boundaries. Visual Cognition, 10, 875-893.

Hart, R. y Moore, G. (1973). The development of spatial cognition: a review. En R. Downs, D. Stea, D. Clark-Carter, A. Heyes y C. Howarth (eds.) (1986), Image and environment: cognitive mapping and spatial behaviour (pp. 246-288). Chicago: Aldine.

Hart, R. y Conn, M. (1991). Developmental perspectives on decision making and action in environments. En T. Garling y G. Evans (eds.) (s. f.), Environment, cognition and action: an integrated approach (pp. 277-294). Nueva York: Plenum Press.
Healy, S. (1998). Spatial representation in animals. Oxford: Oxford University Press.

Hill, E., Rieser, J., Hill, M., Halpin, J. y Halpin, R. (1993). How people with visual impairments explore novel spaces-strategies of good and poor performers. Journal of Visual Impairment and Blindness, 87, 295-301.

Hommel, B. (2005). Perception in action: multiple roles of sensory information in action control. Cognitive Process, 6, 3-14.

Intraub, H. (2004). Anticipatory spatial representation of 3D regions explored by sighted observers and a deaf-andblind-observer. Cognition, 94, 19-37.

Jacobs, L. (1995). The ecology of spatial cognition: adaptive patterns of hippocampal size and space use in wild rodents. En E. Alleva, A. Fasolo, H. Lipp y L. Nadel (eds.) (s. f.), Studies of the brain in naturalistic settings (pp. 301-322), Dordrecht, Países Bajos: Kluwer Academic.

Jacobson, R. (1998). Cognitive mapping without sight: four preliminary studies of spatial learning. Journal of Environmental Psichology, 18, 289-305.

Johnson-Laird, P. (1983). Mental models. Cambridge: Harvard University Press.

Kamil, A. (1994). A synthetic approach to the study of animal intelligence. En L. Real (ed.) (s. f.), Behavioral mechanisms in evolutionary ecology (pp. 11-45). Chicago: University of Chicago Press.

King, J., Burgués, N., Hartley, T., VarghaKhadem, F. y O'Keefe, J. (2002). Human hippocampus and viewpoint dependence in spatial memory. $\mathrm{Hi}^{-}$ рросатрus, 12, 811-820. 
Kitchin, R. (1994). Cognitive maps: what are they and why study them? Journal of environmental Psychology, 14, 1-19.

Klatzky, R. y Lederman (2003). Representing spatial location and layout from sparse kinesthetic contacts. Journal of Experimental Psychology, 2(29), 310-325.

Klaztky, R. (1997). Allocentric and egocentric spatial representations: definitions, distinctions and interconnections. Documento para la Conference on Raumkognition, Trier, Alemania.

Kosslyn, S. (1994). Image and brain-the resolution of the imagery debate. Cambridge: MIT Press.

Lahav, O. y Mioduser, D. (2001). A blind person's cognitive mapping of new spaces using a haptic virtual environment. Journal of Research in Special Educational Needs, 3(3), 172-177.

Loomis, J., Klatzky, R., Golledge, R., Cicinelli, J., Pellegrino, J. y Fry, P. (1993). Nonvisual navigation by blind and sighted: assessment of path integration ability. Journal of Experimental Psychology, 122, 73-91.

Loomis, J., Lippa, Y., Golledge, R. y Klatzky, R. (2002). Spatial updating of locations specified by 3D sound and spatial language. Journal of Experimental Psychology: Learning, Memory \& Cognition, 28, 335-345.

López, O., Sanabria, L., Sarmiento, L., Ibáñez, J. y Valencia, N. (2005). La representación espacial en invidentes congénitos con apoyo de un dispositivo mecatrónico. Bogotá: Universidad Pedagógica Nacional-Colciencias.

Maldonado, L. (2001). Análisis de protocolos: posibilidad metodológica para el estudio de procesos cognitivos. Bogotá: Universidad Pedagógica Nacional.

Millar, S. (1994). Understanding and representing space: theory and evidence from studies with blind and sighted children. Oxford: Clarendon PressOxford University Press.

Montero, I. y León, O. (2002). Clasificación y descripción de las metodologías de investigación en psicología. International Journal of Clinical and Health Psychology, 3(2), 503-508.

O'Keefe, J. y Nadel, L. (1978). The hippocampus as a cognitive map. Oxford: Clarendon Press.

Oakley, I., McGee, M., Brewster, S. y Gray, P. (2000). Putting the feel in look and feel. En Summary Proceedings of ACM CHI'00. Redmond: ACM Press.

Ochaíta, E. y Huertas, J. A. (1993). Spatial representation by people who are blind: a study of the effects of learning and development. Journal of Visual Impairment and Blindness, $87,37-41$.

Posner, M. y DiGirolamo, G. (1998). Executive attention: conflict, target detection, and cognitive control. En R. Parasuraman (ed.) (s. f.), The attentive brain. Cambridge: MIT Press.

Rieser, J., Guth, D. y Hill, E. (1986). Sensitivity to perspective structure while walking without vision. Perception, 15, 173-188.

Rinck, M., Williams, P., Bower, G. y Becker, E. (1996). Spatial situation models and narrative understanding: some generalizations and extensions. Discourse Processes, 21, 23-55.

Roche, R., Mangaoang, M., Commins, S. y O'Mara, S. (2005). Hippocampal contributions to neurocognitive mapping in humans: a new model. Hippocampus, 15, 622-641. 
Sarmiento, L. (2003). Ayudas aumentativas en discapacitados visuales para la representación espacial utilizando las tecnologías de la información y las comunicaciones. Tesis para optar al título de Maestría en Tecnologías de la Información aplicadas a la educación. Bogotá: Universidad Pedagógica Nacional.

Scott, M. (2000). Computational perceptual attention. Disertación para optar por el título de Doctorado en Filosofía, Universidad de Texas, Austin.

Shelton, A. y Gabrieli, J. (2002). Neural correlates of encoding space from route and survey perspectives. $\mathrm{Neu}$ roscience, 22, 2711-2717.

Shettleworth, S. (1998). Cognition, evolution and behavior. Oxford: Oxford University Press.

Sholl, M. (1996). From visual information to cognitive maps. En J. Portugali (ed.) (s. f.), The construction of cognitive maps (pp. 157-186). Dordrecht, Países Bajos: Kluwer Academic.

Stevens, A. y Coupe, P. (1978). Distortions in judged spatial relations. Cognitive Psychology, 10, 422-437.

Tolman, E. (1948). Cognitive maps in rats and men. In: Psychological Review, 55, 189-208.
Ungar, S., Blades, M. y Spencer, C. (1997). Strategies for knowledge acquisition from cartographic maps by blind and visually impaired adults. The Cartographic Journal, 34, 93-110.

Ungar, S., Blades, M., Spencer, C. y Morsley, K. (1994). ¿Can visually impaired children use tactile maps to estimate directions? Journal of Visual Impairment \& Blindness, 88 221-233.

Ungar, S., Simpson, A. y Blades, M. (2004). Strategies for organizing information while learning a map by blind and sighted people. M. Heller y S. Ballasteros (eds.) (s. f.), Touch, blindness and neuroscience. Madrid: Universidad Nacional de Educación a Distancia.

Wang, R. y Spelke, E. (2000). Updating egocentric representations in human navigation. Cognition, 77, 215-250.

Waxman, A., Seibert, M., Bernardon, A. y Fay, D. (1993). Neural systems for automatic target learning and recognition. Lincoln Laboratory Journal, 6, 77-116.

Zwaan, R. y Radvansky, G. (1998). Situation models in language comprehension and memory. Psychological Bulletin, 123, 162-185. 